\title{
The Early Universe, the Present Universe
}

\author{
A. Buonanno
}

\begin{abstract}
Almost ten years ago Gasperini and Veneziano proposed a new picture of the very primordial Universe $(z \gg 1)$ based on string theory, called the pre-big-bang scenario. Here we review the key ideas of this model, its main phenomenological consequences, and the most striking differences with respect to ordinary inflationary models. The second part of this Proceedings is concerned with cosmology at much lower redshifts, $z<2$. We tackle the problem of the motion of inspiraling and merging black-hole binaries, which are among the most promising astrophysical sources of gravitational waves. We discuss a new approach for the two-body problem in general relativity, which makes it possible to study the transition between the adiabatic inspiral and the plunge, and which provides a first estimate of the gravitational waveform emitted during the late dynamical evolution of a binary black-hole system.
\end{abstract}

\section{A scenario for the very primordial Universe from string theory}

Friedman-Robertson-Walker (FRW) cosmological solutions diverge when extrapolated backward in time, raising the so-called singularity problem [1]. Moreover, if the entire history of the universe follows a FRW solution, it is impossible to explain the degree of homogeneity and flatness of our present visible universe. These conundrums are known as the standard cosmological problems [1]; until now, the most credible solution to them assumes that soon after its birth the universe underwent an inflationary phase [1]. Yet inflationary models, still have to deal with the initial-singularity problem, with the problem of the naturalness of initial conditions and the issue of describing the universe at high energy and/or strong coupling. If we had a particle theory capable of describing this initial cosmological phase, then some questions would come naturally: what was before such high-energy stage? Could the origin of time and the issue of initial conditions be decoupled from the singularity problem?

Superstring theory/M-theory [2] is currently considered the most promising extension of the standard model of particle physics. It is the only theory that can unify quantum mechanics and general relativity. If string theory describes our real world, then we should expect that it contains the solution to the cosmological conundrums that we just mentioned. Various attempts have been made in the literature to apply string theory to cosmology. Some results were obtained within M-cosmology, or within its much better understood low-energy limits, such as 11-D supergravity $[3,4]$. Progresses in nonperturbative string theory have been made but until now only with classical solutions that respect a large number of supersymmetries [4]. More recently, interesting new ideas came from models with extra dimensions [5]. 
At low energy, string theory does not give only Einstein general relativity. In the simplest case it leads to the four-dimensional action

$$
\Gamma_{\mathrm{eff}}=\frac{1}{\lambda_{s}^{2}} \int d^{4} x \sqrt{|g|} e^{-\varphi}\left[\mathcal{R}+g^{\mu \nu} \partial_{\mu} \varphi \partial_{\nu} \varphi-\frac{1}{12}(d B)^{2}\right],
$$

where $\varphi$ is the dilaton field, related to the string coupling by $g^{2}=e^{\varphi} ; d B=$ $\partial_{\mu} B_{\nu \rho}+\partial_{\nu} B_{\rho \mu}+\partial_{\rho} B_{\mu \nu}$, where $B_{\mu \nu}$ is the two-form gauge field or antisymmetric field; and where $\lambda_{s}$ is the string scale. In writing Eq. (1) we disregard for simplicity the internal dimensions, whose dynamics can be described in terms of moduli fields [3]. [Henceforth, we pose $\hbar=1$.]

It was realized long ago that potential-driven inflationary scenarios cannot be implemented in string theory if the dilaton field is simply identified with the inflaton field [6]. This result forced people to conceive new ways of reconciling inflation and string theory. Henceforth, we shall discuss one of those attempts, the so-called pre-big-bang (PBB) scenario, originally proposed by Gasperini and Veneziano [7,8].

In the homogeneous and isotropic limit with $B=0\left[d s^{2}=-d t^{2}+a^{2}(t) d \boldsymbol{x}^{2}, \varphi=\right.$ $\varphi(t)$ ], the solution of the low-energy string-effective action (1) satisfies the scalefactor-duality (SFD) symmetry: $a(t) \rightarrow 1 / a(t), \varphi(t) \rightarrow \varphi(t)-6 \log a(t),{ }^{1}$ with $a(t) \sim t^{1 / \sqrt{3}}$ and $\varphi(t) \sim-\log t$. Deducing this result Veneziano [7] conceived the idea, subsequently sharpened by Gasperini and Veneziano [8], of implementing the inflationary phase at times before the would-be big-bang singularity. Indeed, it is easily shown that for $t<0, \dot{a}>0, \ddot{a}>0$; that is the universe undergoes a (super) inflationary phase! Two different but physically equivalent descriptions of the PBB phase exist: either in the string-frame picture given by Eq. (1), where the universe undergoes an accelerated expansion $(H>0, \dot{H}>0, \dot{\varphi}>0)$, or in the Einstein-frame picture, where the action has the standard Hilbert-Einstein form and the evolution of the universe is described by an accelerated contraction, or gravitational collapse $(H<0, \dot{H}<0, \dot{\varphi}>0)$.

This radically new kind of inflation is driven by the kinetic energy of the dilaton field and forces both the string coupling $(\dot{g}>0)$ and the spacetime curvature to grow toward the future. As a consequence, at least in the homogeneous case, the inflationary stage lasts for ever $(t \rightarrow-\infty)$ and the initial state of the universe is nearly flat, cold and decoupled: $g \ll 1, \mathcal{R} \lambda_{s}^{2} \ll 1$. At some later time, evolving toward the would-be big-bang singularity, the universe enters a phase of high curvature $\left(\mathcal{R} \lambda_{s}^{2} \sim 1\right)$ and/or strong coupling $(g \sim 1)$, where the perturbative description of the PBB phase breaks down and higher order corrections to the low-energy string effective action (1) should be taken into account:

$$
\begin{aligned}
\Gamma_{\text {eff }}= & \frac{1}{\lambda_{s}^{2}} \int d^{4} x \sqrt{|g|} e^{-\varphi}\left\{\mathcal{R}+g^{\mu \nu} \partial_{\mu} \varphi \partial_{\nu} \varphi+\cdots+\alpha^{\prime}\left[\mathcal{R}^{2}+(\partial \varphi)^{4}+\cdots\right]\right. \\
& \left.+e^{\varphi}\left[\mathcal{R}+(\partial \varphi)^{2}+\alpha^{\prime}\left(\mathcal{R}^{2}+(\partial \varphi)^{4}+\cdots\right)\right]+\cdots\right\}
\end{aligned}
$$

\footnotetext{
${ }^{1}$ Here for convenience the origin of time has been fixed at $t=0$.
} 
where $\alpha^{\prime}=\lambda_{s}^{2}$ governs the finite-string-size effects, and $g=e^{\varphi}$ governs the quantum loop corrections. The issue of connecting the perturbative and nonperturbative PBB phases to the FRW cosmologies of radiation and matter eras has been called the graceful exit problem. Various analytical and numerical investigations have been made including first order finite-string-size effects and/or quantum loop corrections [9]. A complete solution of the graceful exit problem is still not available.

In Fig. 1 we draw the evolution of the Hubble expansion rate $H=\dot{a} / a$ in the homogeneous PBB cosmology. The central blob refers to the high-curvature and/or strong-coupling phase.

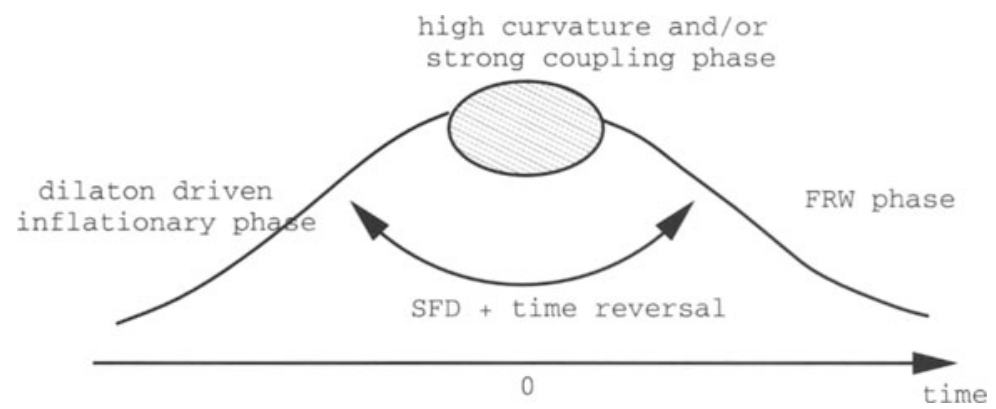

Fig. 1. Evolution in time of the Hubble expansion rate $H=\dot{a} / a$ in the homogeneous PBB cosmology

It was shown that to solve the standard cosmological problems of homogeneity and flatness in PBB cosmology, we must require that initially the string coupling $g_{\text {in }}<10^{-26}$ and that $H_{\text {in }} \lambda_{s}<10^{-19}$ [7,10,11]. Quasi-homogeneous cosmological solutions in the perturbative inflationary phase of the PBB scenario have been derived $[11,12]$ by applying the spatial-gradient expansion technique. Because of the presence of the dilaton field in the action (1), the Belinskii-Khalatnikov-Lifshitz (BKL) oscillations can last at most for a finite time [13]. Afterwards, the universe enters an era where spatial gradients become less and less important as we move toward the singularity; that is $\left(\nabla_{\text {spatial }} \varphi\right)^{2} / \dot{\varphi}^{2}, R / \dot{\varphi}^{2} \rightarrow 0$ as $t \rightarrow 0^{-}$. However, recently it was pointed out [14] that this result is somewhat spoiled when other $p$-form fields (including the antisymmetric field B) are present in the low-energy string-effective action (1). Their presence leads to the generic appearance of an inhomogeneous chaos near the would-be big-bang singularity, ultimately leading to a string-scale foam [14].

As we anticipated, one of the most striking differences between the PBB scenario and the standard cosmological models is the description of the initial state of the universe. In the PBB model the universe is initially in a weakly-coupled, classical state, consisting of a stochastic bath of gravitational and dilatonic waves [15]. By the mechanism of gravitational instability, this state can give rise to our universe (modulo the assumption of a graceful exit from the PBB phase to the standard FRW era). Indeed, if those initial waves satisfy a certain strength criterion [15], viewed 


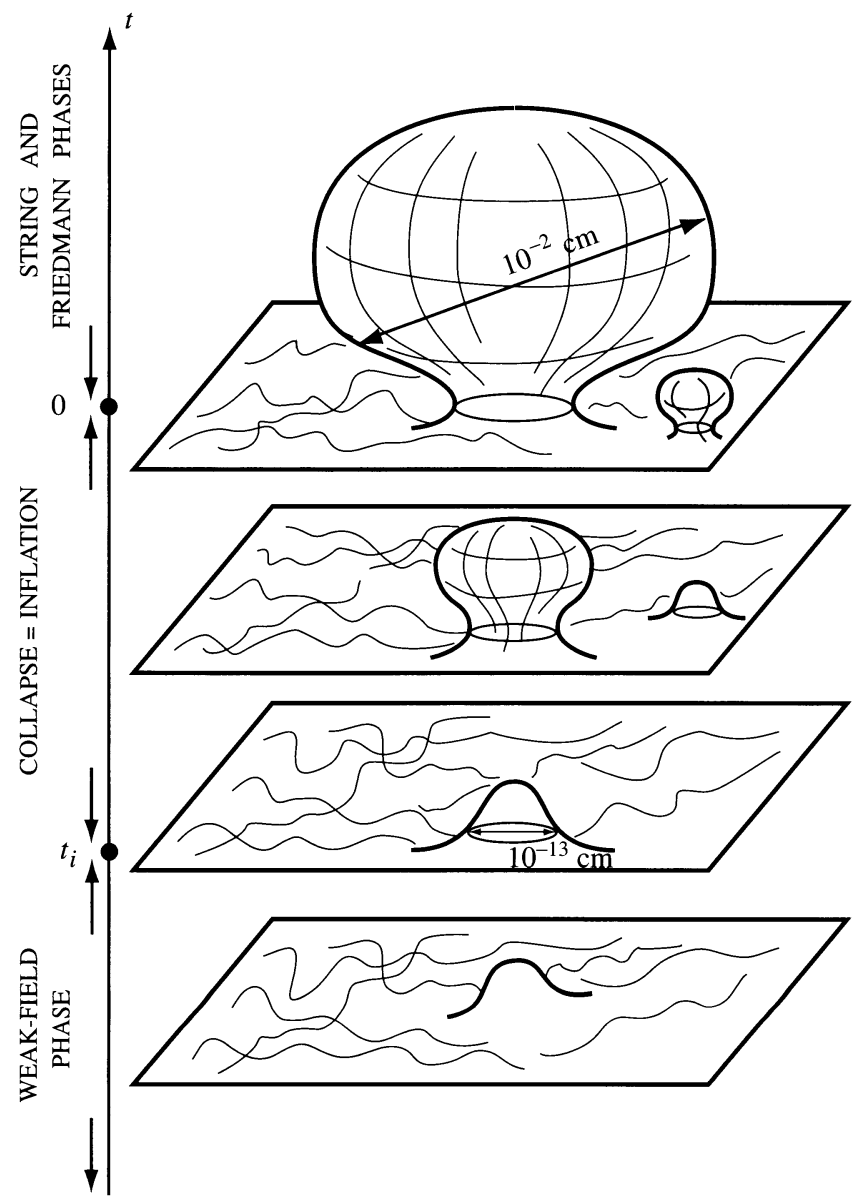

Fig. 2. Naive evolution of a PBB bubble of initial size $1 \mathrm{fm}$, which originated from a classical fluctuation in the initial bath of gravitational and dilatonic waves. We follow the PBB bubble up to the would-be big-bang singularity hypersurface, where its size is on the order of $0.1 \mathrm{~mm}$ (this is the right size to explain the present scale of homogeneity of the universe)

in the Einstein frame, they collapse but viewed in the physically more appropriate string frame, each gravitational collapse leads to the local birth of a baby inflationary universe. It was then claimed [15] that the occurrence of a PBB inflation period is as generic in string theory as the occurrence of gravitational collapse in general relativity. In Fig. 2 we draw the evolution of a PBB bubble, from its inception as classical fluctuation of the initial sea of dilatonic and gravitational waves, up to the beginning of the FRW era.

The stochastic version of PBB cosmology was originally intended to address some concerns about fine-tuning $[10,16]$ in the PBB scenario. It can be shown $[11,12,15]$ 
that the only condition needed for the birth of a PBB bubble of size $\mathrm{H}^{-1}$ (in string units), is similar to the corresponding condition in chaotic inflation [1]. Namely, the inhomogeneous contributions to the local Friedmann equation should be fractionally small (say by a factor of five) compared to the homogeneous contribution, $\dot{\varphi}^{2} \sim H^{2}$. However, it has been recently realized that the PBB scenario is not very effective in smoothing out the initial tensor classical inhomogeneities [17]. As a consequence, if we wish that generic coarsely homogeneous bubbles evolve into our universe, we need to require that $g_{\text {in }} \lesssim 10^{-35}$, that is the initial value of the string coupling should be parametrically smaller than the minimal value $g_{\text {in }}^{\min } \simeq 10^{-26}$ needed to solve the standard cosmological conundrums mentioned earlier.

Significant effort has been spent on extracting the observational predictions of the PBB scenario. During the dilaton-driven inflationary phase, the kinetic energy of the dilaton field is converted into particles, by the well known mechanism of amplification of quantum vacuum fluctuations. This phenomenon is also present in ordinary inflationary models - for example it is supposedly responsible of the inhomogeneities in the Cosmic Microwave Background Radiation (CMBR). Due to the richness of the particle content of string theory (axions, scalar fields, gauge fields,...), and due to the nontrivial coupling (depending also on the compactification of internal dimensions) between those fields and the background fields (i.e., the dilaton, moduli and gravitational field), a much larger number of species can be produced out of vacuum in the PBB scenario than in ordinary inflationary models. It is interesting to note that at second order in perturbation theory the fluctuation $\psi$ of whatever field is governed by the action [written in the conformal time $\eta$ $(d t=a d \eta)][18]$

$$
\delta \Gamma_{\mathrm{eff}}=\int d \eta d^{3} x \tilde{a}_{\psi}^{2}(\eta)\left[\left(\frac{\partial \psi}{\partial \eta}\right)^{2}-(\nabla \psi)^{2}\right],
$$

where the only dependence on the specific field comes through the function $\tilde{a}_{\psi}$, the so-called pumping field. For example, for gravitons, for scalar fields and for moduli fields, we find $\tilde{a}=a e^{-\varphi / 2}[18-20]$. If we assume static internal dimensions, then gauge fields have $\tilde{a}=e^{-\varphi / 2}[18,21]$, while for axion fields $\tilde{a}=a e^{\varphi / 2}[18,22]$. Contrary to what happens in potential-driven inflation, in PBB cosmology the spectrum of the energy density versus frequency can grow, decrease or be constant. More specifically the PBB model predicts a stochastic background of gravitational waves whose energy-density spectrum increases at very low frequencies [19], and whose amplitude might be well above that predicted by models of standard inflation at frequencies $\sim 100 \mathrm{~Hz}$, just in the band of best sensitivity for future earth-based gravitational-wave interferometers, such as the Laser Inteferometric Gravitational Wave Observatory (LIGO) and VIRGO [23]. In certain regions of the free-parameter space, quantum vacuum electromagnetic fluctuations, amplified by the nonconformal coupling between gravitational and electromagnetic fields, could produce the primordial seeds responsible for the formation of galactic and extragalactic magnetic fields [21]. However, despite the efforts to investigate the amplification of axion quantum fluctuations $[18,22,24]$ (which can produce a nearly constant energy-density 
spectrum versus frequency), the PBB scenario still lacks a mechanism for describing the inhomogeneities in the CMBR, and the formation of large scale structures.

The mechanism of reheating in PBB cosmology has also been investigated [20]. Let us first note that in standard cosmological models the inflationary era is dominated by potential energy; the post-inflationary phase is driven by inflaton condensates, that later on, decay into radiation (in the reheating process) giving rise to the birth of the hot big bang. By contrast, as discussed above, in the PBB scenario it is the kinetic energy of the dilaton field which drives the inflationary phase. Therefore, to explain the birth of the hot big-bang era in the PBB model, it was originally suggested [25] that the particles present at the very beginning of the radiation era could have originated from the mechanism of quantum-vacuum fluctuations during the PBB phase. However, it was found [20] that PBB models inevitably face a severe gravitino/moduli problem. Indeed, they predict quite generically that at the beginning of the radiation era, the moduli and gravitinos, produced gravitationally or from scattering processes of the thermal bath, will have a number-density to entropy-density ratio that is far in excess of the big-bang-nucleosynthesis bound. Hence, in the PBB scenario, reheating cannot be implemented solely by gravitational production. Depending on the details of the transition from PBB era to FRW phase, late-entropy production to the level of $\Delta s \gtrsim 10^{5}-10^{10}$ is mandatory to dilute those dangerous relics. This entropy production can be viewed as a period of secondary reheating; that is as the real birth of the hot big-bang era in the PBB scenario. Sufficient entropy can be produced by the domination and decay of the zero-mode of a modulus field with mass $\sim 10^{6} \mathrm{GeV}$; this could well be the dilaton field, initially displaced from the minimum of its potential by an amount on the order of the string mass $M_{s} \sim 10^{18} \mathrm{GeV}$ [26]. Nevertheless, the above source of entropy comes with a bonus: baryogenesis can be implemented in a natural way via the so-called Affleck-Dine mechanism [27]. Finally, we notice that in PBB cosmology typical reheating temperatures vary in the range $T_{\mathrm{RH}} \sim 1-10^{5} \mathrm{GeV}$.

In conclusion, the PBB scenario is certainly an interesting attempt of reconciling string theory and cosmology. It has proposed a new, elegant way to implement inflation, which is based on a duality symmetry of string theory and uses the kinetic energy of the dilaton field; it has proposed the rather unconventional idea of decoupling the singularity problem from the issue of initial conditions, by assuming that the universe originated from a classical, weakly coupled state; it has pointed out the rich variety of energy-density spectra of particles produced out of vacuum during the PBB inflationary phase, whose details strongly depend on the background dynamics of dilaton, moduli and gravitational field.

\section{Coalescing compact binaries: a new approach to the two-body problem in general relativity}

Binary systems made of compact objects (neutron stars or black holes) that inspiral toward coalescence because of gravitational-radiation damping are among the most promising candidate sources for interferometric gravitational-wave $(\mathrm{GW})$ de- 
tectors, such as the Laser Inteferometric Gravitational Wave Observatory (LIGO) and VIRGO [23]. One of the most important issue in gravitational-wave research is the generation problem [28]; that is, the link between the radiative transverse traceless (TT) gravitational field $h_{i j}^{\mathrm{TT}}$, far away from the source, and the motion of the source. This link is provided at lowest order in the post-Newtonian (PN) expansion by the Einstein's quadrupole formula [28], which gives for the radiative field:

$$
h_{i j}^{T T}(T, D)=\frac{2 G}{c^{4} D} \mathcal{P}_{i j k m}(N) \frac{d^{2}}{d T^{2}} Q_{k m}\left(T-\frac{D}{c}\right),
$$

where $Q_{i j}(i, j=1,2,3)$ is the tracefree quadrupole moment of the source; $D$ is the distance from the source; $N=X / D$ is the unit vector from the source to the observer; $\mathcal{P}_{i j k m}(N)$ is the TT projection operator onto the plane orthogonal to $N ; G$ is the Newton constant; and $c$ is the speed of light.

The inspiral waveform enters the detector band during the last few minutes of evolution of the binary. The LIGO/VIRGO community plans to track the signal phase and build up the signal-to-noise ratio by integrating the signal for the time during which it stays in the detector band. This is achieved by filtering the detector output with a template which is an (approximate) copy of the exact, observed signal. From Eq. (4) (and its extensions at higher PN orders) we see that the more precisely we know the two-body motion, the more accurately the PN template $h_{i j}^{\mathrm{TT}}$ will describe the exact gravitational waveform.

Henceforth, our analysis will be restricted to nonspinning black holes. In Fig. 3 we show a typical gravitational waveform. The part of the waveform drawn with a continuous line is emitted during the inspiral phase when the two black holes are largely separated $(r \geq 10 M)$. We denoted by $r$ the radial separation and by $M$ the total mass of the binary system. During the inspiral, the two black holes follow an adiabatic sequence of quasi-circular orbits. The equation of motion in the center-ofmass frame can be written schematically as [28]

$$
\frac{d^{2} \boldsymbol{x}}{d t^{2}}=-\frac{G M \boldsymbol{x}}{r^{3}}\left[1+\mathcal{O}(\epsilon)+\mathcal{O}\left(\epsilon^{2}\right)+\mathcal{O}\left(\epsilon^{5 / 2}\right)+\cdots\right] \times[1+\mathcal{O}(v)+\cdots],
$$

where $\boldsymbol{x}$ denotes the separation vector between the two bodies and $r=|\boldsymbol{x}|$. Equation (5) is characterized by a double expansion: in the PN parameter $\epsilon \sim v^{2} / c^{2} \sim M / r$, and in the parameter $v=m_{1} m_{2} / M^{2}$, where $m_{1}$ and $m_{2}$ are the masses of the two black holes. The parameter $v$ ranges between 0 (test-mass limit) and 1/4 (equal-mass case).

The PN expansion converges badly: as the two bodies draw closer, it becomes more and more difficult to extract nonperturbative information from the PN series. Specifically, when the distance between the inspiraling black holes shrinks to $r \lesssim 10 M$, the PN expansion can no longer be trusted [29]. The dashed line in Fig. 3 depicts the part of the waveform emitted during the final phase of evolution, when nonlinearities and strong curvature effects become important. During this stage the PN expansion fails and nonperturbative analytical and/or numerical techniques should be used. This final phase includes the transition from the adiabatic inspiral to the plunge, beyond 


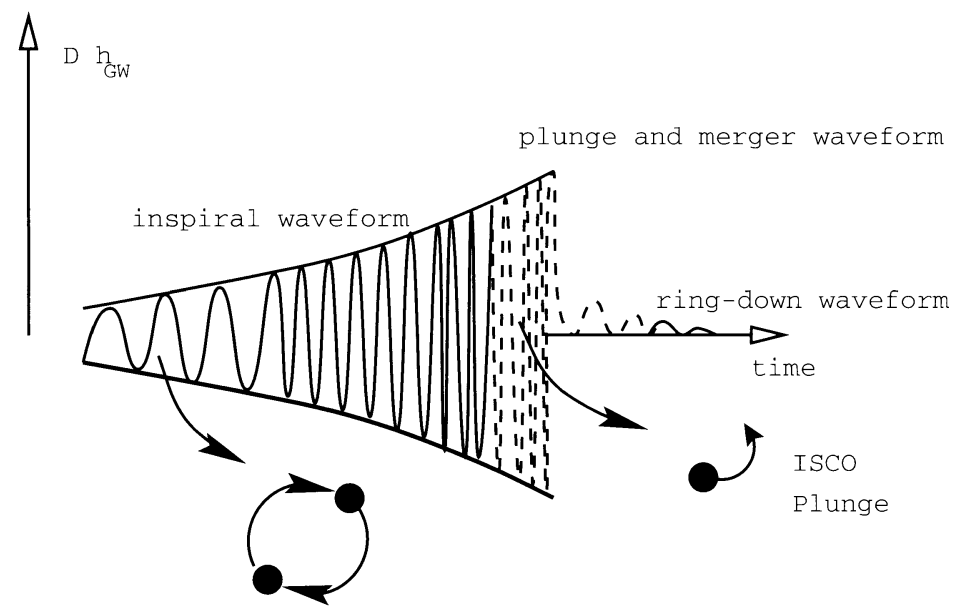

Fig. 3. Typical gravitational waveform emitted throughout the inspiral, plunge and ringdown phase

which the two-body motion is driven (almost) only by the conservative part of the dynamics. The plunge starts at the innermost stable circular orbit (ISCO) of the binary black holes. Due to the failure of the PN expansion, different predictions for the ISCO location have been provided so far in the literature [30,31]. Beyond the plunge the two black holes merge, forming a Kerr black hole. As the system reaches the stationary Kerr state, the nonlinear dynamics of the merger resemble more and more the oscillations of the black-hole quasi-normal modes [32]. During this phase, often called the ring-down phase, the gravitational signal will be a superposition of exponentially damped sinusoids.

It seems likely that the first detection of gravitational waves with LIGO and VIRGO interferometers will come from binary systems made of massive black holes of comparable masses, say with a total mass $M \simeq 15 M_{\odot}+15 M_{\odot}$. If we restrict our attention to nonspinning black holes, it is easily shown that the gravitational-wave frequency at the ISCO for such massive systems is very close (not accidentally!) to the location of the minimum for the detector's noise spectral density. For example, for the first generation of LIGO interferometers, the maximum of the signal-to-noise ratio for nonspinning black holes of total mass $M \simeq 15 M_{\odot}+15 M_{\odot}$ is reached at $f_{\text {detection }} \simeq 167 \mathrm{~Hz}$, which is quite close to $f_{\mathrm{GW}}^{\mathrm{ISCO}} \simeq 180 \mathrm{~Hz}$. Therefore, for data analysis purposes it is quite desirable to have a thorough knowledge of the late dynamical evolution of comparable-mass binaries.

Despite the progress made by the numerical relativity community during the recent years, an estimate of the complete waveform emitted by a black-hole binary with comparable masses has not yet been provided. Preliminary results for the plunge, merger and ring-down waveform were only recently obtained [33]. To tackle the delicate issue of the late dynamical evolution, Buonanno and Damour introduced a new nonperturbative analytical approach to study the motion of two nonspinning 
bodies in general relativity $[31,34]$. This approach should be able to capture the crucial features of the transition from the adiabatic inspiral to the plunge. Henceforth, we shall refer to this new technique as effective-one-body (EOB) approach.

The EOB approach combines two PN resummation techniques. The first method [31], inspired by an approach introduced by Brézin, Itzykson and ZinnJustin [35] to study electromagnetically interacting two bodies, makes it possible to derive a nonperturbative estimate for the conservative part of the nonlinear force law that governs the motion of comparable-mass binaries. The basic idea [31], illustrated in Fig. 4, is to map the real conservative two-body dynamics up to $2 \mathrm{PN}$ order (see below for the extension at 3PN order) onto an effective one-body problem, where a test particle of mass $m_{0}$ moves in some effective background metric $g_{\mu \nu}^{\text {eff }}$. This mapping

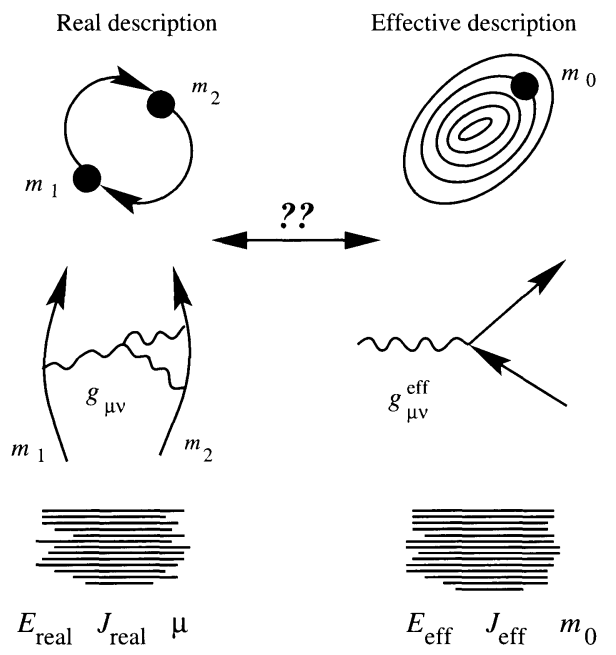

Fig. 4. How the EOB approach matches the real two-body problem (on the left) and the effective one-body problem (on the right) in general relativity

has been worked out within the Hamilton-Jacobi formalism, imposing that the adiabatic invariants of the real and effective description coincide $J_{\text {real }}=J_{\text {eff }}, \mathcal{I}_{\text {real }}=\mathcal{I}_{\text {eff }}$, where $J$ denotes the total angular momentum, and $\mathcal{I}$ the radial action variable. While doing so, we allow a transformation of the energy axis, $E_{\text {real }}=f\left(E_{\text {eff }}\right)$, where $f$ is a generic function. The test mass $m_{0}$ in the effective description was assumed to be equal to the reduced mass $\mu=m_{1} m_{2} / M$ of the two-body system.

After applying the rules to define the mapping, we found that, as long as radiationreaction effects are not taken into account, the effective metric is just a deformation of the Schwarzschild metric, with deformation parameter $v=\mu / M$. The effective 
metric reads [31]

$$
\begin{aligned}
& d s_{\mathrm{eff}}^{2}=-A(R) c^{2} d t^{2}+\frac{D(R)}{A(R)} d R^{2}+R^{2} d \Omega^{2} \\
& A(R)=1-2 \frac{G M}{c^{2} R}+2 v\left(\frac{G M}{c^{2} R}\right)^{3}, \quad D(R)=1-6 v\left(\frac{G M}{c^{2} R}\right)^{2}
\end{aligned}
$$

The effective and real (nonrelativistic) energies are related by [31]

$$
\frac{E_{\mathrm{eff}}^{\mathrm{NR}}}{m_{0} c^{2}}=\frac{E_{\mathrm{real}}^{\mathrm{NR}}}{\mu c^{2}}\left(1+\frac{\nu}{2} \frac{E_{\mathrm{real}}^{\mathrm{NR}}}{\mu c^{2}}\right)
$$

Remarkably, this mapping between the real and the effective nonrelativistic energies coincides with the mapping obtained by Brézin, Itzykson and Zinn-Justin [35] in the context of quantum electrodynamics, where these authors mapped the one-body relativistic Balmer formula onto the two-body energy formula.

The EOB approach provides a method to resum nonperturbatively the badly convergent $\mathrm{PN}$-expanded dynamics of the real description. Indeed, it gives the following improved real Hamiltonian [34]:

$$
H_{\text {real }}^{\text {improved }}=M c^{2} \sqrt{1+2 v\left(\frac{H_{\mathrm{eff}}^{v}-\mu c^{2}}{\mu c^{2}}\right)},
$$

where

$$
H_{\mathrm{eff}}\left(\nu, R, P_{R}, P_{\varphi}\right)=\mu c^{2} \sqrt{A(R)\left(1+\frac{A(R) P_{R}^{2}}{\mu^{2} c^{2} D(r)}+\frac{P_{\varphi}^{2}}{\mu^{2} c^{2} R^{2}}\right)} .
$$

The basic idea that underlies the mapping of two-body general relativistic dynamics onto an effective one-body problem, was recently extended to classical electrodynamics to test its robustness. Reference [36] discussed the mapping of the conservative part of two-body electrodynamics (i.e., of a two-body system of charges $e_{1}$ and $e_{2}$ with $e_{1} e_{2}<0$ ) onto the dynamics of a test particle of charge $e_{0}$ moving in some external electromagnetic field (see Fig. 5); the author took into account recoil effects and relativistic corrections up to second post-Coulombian order. In this case the expansion parameter is the classical radius $\alpha_{0} / m_{0} c^{2}$, where $\alpha_{0}=e_{0}^{2}$. Unlike the results obtained in general relativity, in classical electrodynamics it is not possible to implement the matching without introducing external parameters in the effective electromagnetic field. For example, it was found that the effective vector potential $A_{\mu}^{\text {eff }}$ must depend either on the energy or the angular momentum. However, if we relax the assumption that the effective test particle moves in a flat spacetime, then it is sufficient to introduce a scalar potential $\varphi^{\text {eff }}$ to obtain the matching between real and effective descriptions. Let us finally note that even in classical electrodynamics the real and effective nonrelativistic energies are mapped through the same Eq. (6). 
Real description
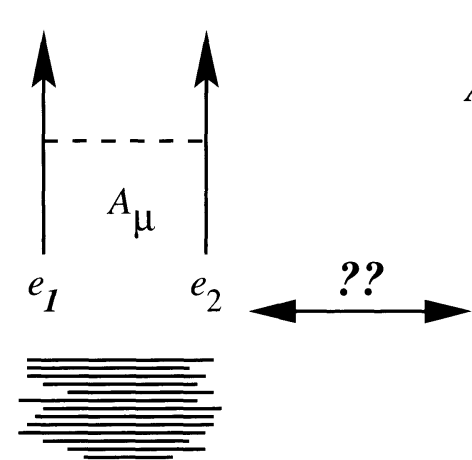

$E_{\text {real }} J_{\text {real }} \alpha$
Effective description
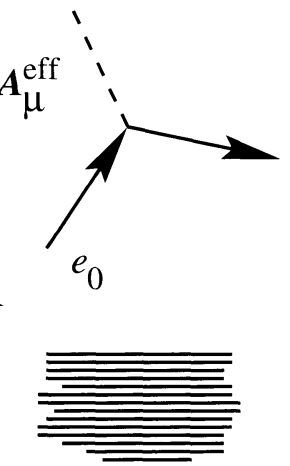

$E_{\text {eff }} \quad J_{\text {eff }} \quad \alpha_{0}$

Fig. 5. Matching between the real two-body problem (on the left) and the effective one-body problem (on the right) in classical electrodynamics

We now go back to the general relativistic case. Earlier we only discussed the conservative part of the dynamics; now let us introduce radiation-reaction effects. Using Padé approximants, Damour, Iyer and Sathyaprakash [37] gave a resummed estimate of the energy-loss rate along circular orbits $\Phi_{\text {circ }}$, up to $2.5 \mathrm{PN}$ order. Buonanno and Damour [34] then combined this resummation method with the EOB approach, and deduced a system of ordinary differential equations which describe the late dynamical evolution of a binary-black-hole system. In spherical coordinates $\left(\varphi, R, P_{\varphi}, P_{R}\right)$, their relevant equations are [34]:

$$
\begin{aligned}
\frac{d R}{d t}=\frac{\partial H_{\mathrm{real}}^{\mathrm{impr}}}{\partial P_{R}}, & \frac{d P_{R}}{d t}+\frac{\partial H_{\mathrm{real}}^{\mathrm{impr}}}{\partial R}=0, \\
\frac{d \varphi}{d t}=\frac{\partial H_{\mathrm{real}}^{\mathrm{impr}}}{\partial P_{\varphi}}, & \frac{d P_{\varphi}}{d t}=-\frac{\Phi_{\mathrm{circ}}}{\dot{\varphi}} .
\end{aligned}
$$

These equations can be used analytically or numerically to study the transition between the adiabatic inspiral and the plunge. Specifically, by a linear expansion in the radial velocity $\dot{R}$, they deduced the following characteristic equation [34]

$$
\frac{d^{3} R}{d t^{3}}+\omega_{R}^{2}(R) \frac{d R}{d t}=-B_{R}(R) .
$$

The quantity $\omega_{R}^{2}$ plays the role of a restoring force. It is the square of the frequency of radial oscillations, and it is proportional to the curvature of the effective radial potential (it vanishes at the ISCO). The quantity $-B_{R}(\propto \nu)$ is a driving force, coming from gravitational radiation damping. The term $d^{3} R / d t^{3}$ is an inertia term, which is neglected in the adiabatic approximation, but should be retained when describing the motion in proximity of the ISCO and beyond it. Ori and Thorne [38] independently 
derived an equation analogous to Eq. (11) for a test particle moving along quasi circular equatorial orbits in Kerr spacetime.

Let us discuss the main features of the transition from inspiral to plunge in the two extreme limits $v \ll 1$ and $v=1 / 4$. The case $v \ll 1$ refers to binary-black-hole systems in which a very small black hole spirals around a supermassive black hole. These are typical GW sources for the future Laser Interferometer Space Antenna (LISA). In this case, the transition from adiabatic inspiral to plunge is sharply localized around the ISCO and various interesting quantities satisfy very simple scaling laws. For example the radial momentum at the ISCO scales like $v^{3 / 5}$, and the number of GW cycles left after the ISCO scales like $v^{-1 / 5}[34,38]$. Ori and Thorne [38] pointed out that likely LISA could observe the transition from inspiral to plunge.

For equal-mass binaries $(v=1 / 4)$, we compare in Fig. 6 the "exact" gravitational waveform, obtained by solving Eqs. (11) numerically, with its adiabatic approximation. Contrary to the case $v \ll 1$, for equal-mass black holes the radiation damping effects become important in an extended region on the order of $\Delta\left(R c^{2} / G M\right) \sim 1$ above the naive (Schwarzschild) ISCO $R=6 G M / c^{2}$. In Fig. 6 the naive ISCO is found at $t \sim 50 \mathrm{M}$. Hence, the transition from inspiral to plunge is rather blurred. Moreover, as Fig. 6 shows, the dephasing between the exact and the adiabatic waveform becomes visible somewhat before the naive ISCO. The plunge part of the exact waveform looks like a continuation of the inspiral part. This happens because the orbital motion remains quasi-circular throughout the plunge.

Recently Damour, Iyer and Sathyaprakash [39] investigated the consequences of the EOB waveform for LIGO/VIRGO data analysis. They found the interesting result that $\mathrm{GW}$ radiation coming from the plunge and merger can significantly enhance the signal-to-noise ratio for binaries of total mass $M \gtrsim 30 M_{\odot}$.

In Fig. 7 we have blown up the plunge and merger part of the waveform shown in Fig. 6, and we have included the ring-down waveform [34]. The ringdown waveform contains only the mode that is damped more slowly, $l=2, m=2$ [32], at frequency $\omega_{q n m} \sim 1880\left(10 M_{\odot} / M_{\mathrm{BH}}\right) \mathrm{Hz}$, where $M_{\mathrm{BH}}$ is the mass of the final hole formed. The dimensionless rotation parameter is $a_{\mathrm{BH}}=J_{\mathrm{BH}} /\left(G M_{\mathrm{BH}}^{2}\right)=0.795$, where we denoted the angular momentum of the final Kerr black hole by $J_{\mathrm{BH}}$. The energy emitted during the plunge is $\sim 0.7 \%$ of $M$, with a comparable energy loss $\sim 0.7 \%$ of $M$ during the ring-down phase. This gives a total energy released of $\sim 1.4 \%$ of $M$ to be contrasted with the much larger value 4-5\% of $M$ recently estimated in [33].

Before closing, let us observe that the EOB approach was extended to the 3PN order by Damour, Schafer and Jaranowski [40]. They found that at the 3PN order the mapping between the effective and the real problem exists only if we abandon the hypothesis (used at 2PN order [31]) that the effective test-mass motion is geodesic. Note also that the relation (11) between the effective and real (nonrelativistic) energies survives at $3 \mathrm{PN}$ order.

In conclusion, we have discussed how analytical resummation techniques can cope with the final nonperturbative phase of binary-black-hole evolution. By reducing the two-body dynamics onto a simpler auxiliary one-body problem [31], and by resumming radiation-reaction effects with Padé approximants [37], we end up with 


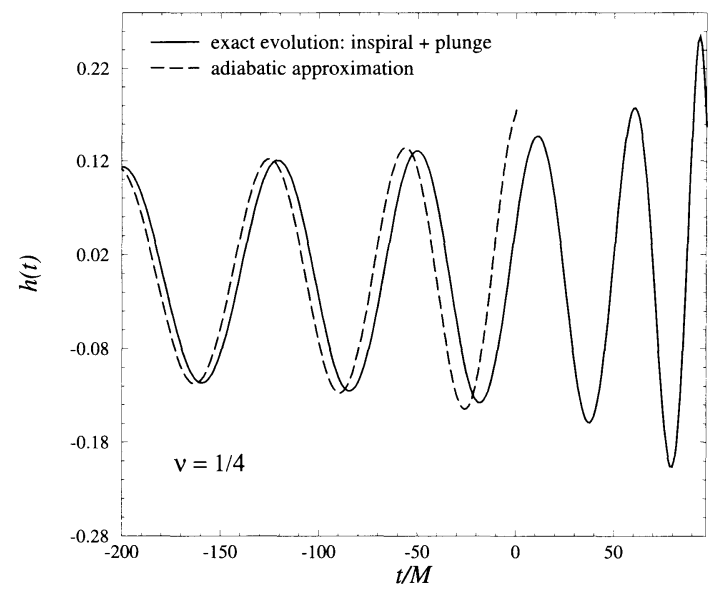

Fig. 6. Comparison between the exact gravitational waveform and the adiabatic waveform. Note that $t \sim-200 M$ and $t \sim 90 M$ corresponds to the radial separations $R \sim 8 G M / c^{2}$ and $R \sim 2.8 G M / c^{2}$, respectively. The naive (Schwarzschild) ISCO $R=6 G M / c^{2}$ is located at $t \sim 50 M$

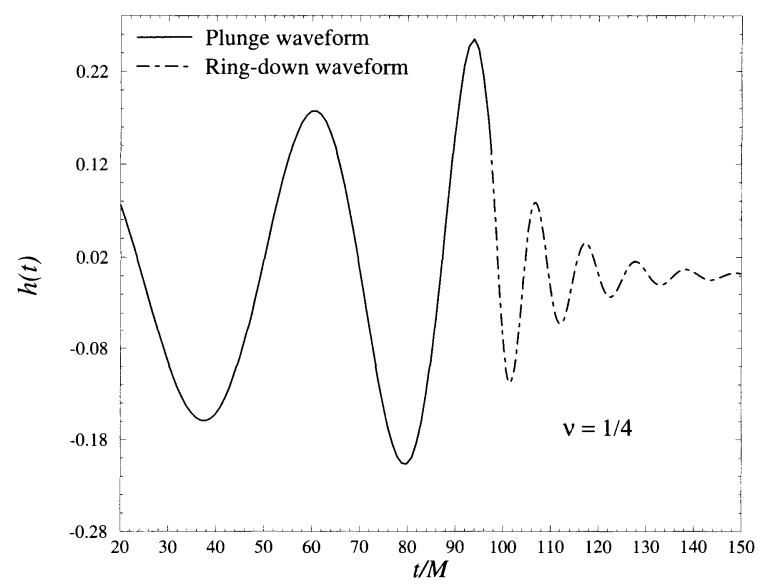

Fig. 7. Plunge and ring-down gravitational waveform obtained from the EOB approach

an explicit analytical system of ordinary differential equations that describes the transition from inspiral to plunge [34]. Beyond the estimation of the gravitational waveform, the most important and urgent application of this approach will be to provide initial dynamical data for numerical relativity investigations, of black holes that have just started their plunge motion. 
Acknowledgements. The author wishes to thank all the people who collaborated with her on these research topics. They deserve much of the merit for the SIGRAV Prize the author received. They are: T. Damour, M. Gasperini, M. Lemoine, M. Maggiore, K. Meissner, K.A. Olive, C. Ungarelli and G. Veneziano.

\section{References}

1. Kolb, E.W., Turner, M.S. (1990): Early cosmology. Reading Massachusetts, Addison Wesley;

Linde, A.D. (1990): Particle physics and inflationary cosmology. Harwood

2. Polchinski, J. (1998): String theory. Cambridge

3. Caroll, S.: Tasi lectures: Cosmology for string theorists; hep-th/0011110

4. Banks, T. (1999): $M$-theory and cosmology, Les Houches 1999 Summer School, $L ' U$ nivers Primordial; hep-th/9911067

5. Antoniadis, I., Arkani-Hamed, N., Dimopoulos, S., Dvali, G. (1998): Phys. Lett. B 429, 263; (1998): Phys. Lett. B 436, 257;

Randall, L., Sundrum, R. (1999): Phys. Rev. Lett. 83, 3370;

(1999): Phys. Rev. Lett. 83, 4690

6. Campbell, B.A., Linde, A.D., Olive, K.A. (1991): Nucl. Phys. B 335, 146;

Brustein, R., Steinhardt, P.J. (1993): Phys. Lett. B 302196

7. Veneziano, G. (1991): Phys. Lett. B 265, 287

8. Gasperini, M., Veneziano, G. (1993): Astropart. Phys. 1, 317; (1993): Mod. Phys. Lett. A 8, 3701

9. Gasperini, M., Maggiore, M., Veneziano, G. (1997): Nucl. Phys. B 494, 315;

Brustein, R., Madden, R. (1997): Phys. Lett. B 410, 110;

(1998): Phys. Rev. D 57, 712;

Foffa, S., Maggiore, M., Sturani, R. (1999): Nucl. Phys. B 552, 395

10. Turner, M.S., Weinberg, E.J. (1997): Phys. Rev. D 56, 4604

11. Buonanno, A., Meissner, K.A., Ungarelli, C., Veneziano, G. (1998): Phys. Rev. D 572543

12. Veneziano, G. (1997): Phys. Lett. B 406, 297

13. Belinskii, V.A., Khalatnikov, I.M. (1970): Sov. Phys. JETP 30, 1174;

Belinskii, V.A., Lifshitz, E.M., Khalatnikov, I.M. (1971): Sov. Phys. Uspekhi 13, 745

14. Damour, T., Henneaux, M. (2000): Phys. Rev. Lett. 85, 920;

(2000): Phys. Lett. B 488 108;

(2000): Erratum Phys. Lett. B 491, 377; hep-th/0012172

15. Buonanno, A., Damour, T., Veneziano, G. (1999): Nucl. Phys. B 543, 275

16. Linde, A.D., Kaloper, N., Bousso, R. (2000): Phys. Rev. D 59, 043508

17. Buonanno, A., Damour, T. (2001): Phys. Rev. D64, 043501; gr-qc/0102102

18. Buonanno, A., Meissner, K., Ungarelli, C., Veneziano, G. (1998): JHEP 9801, 004;

Brustein, R., Hadad, M. (1998): Phys. Rev. D 57, 725

19. Brustein, R., Gasperini, M., Giovannini, M., Mukhanov, S., Veneziano, G. (1995) Phys. Rev. D 51, 6744;

Brustein, R., Gasperini, M., Giovannini, M., Veneziano, G. (1995): Phys. Lett. B 361 45; Buonanno, A., Maggiore, M., Ungarelli, C. (1997): Phys. Rev. D;

Maggiore, M. (2000): Phys. Rep. 331283

20. Buonanno, A., Lemoine, M., Olive, K.A. (2000): Phys. Rev. D 62, 083513

21. Gasperini, M., Giovannini, M., Veneziano, G. (1995): Phys. Rev. Lett. 75, 3796;

Lemoine, D., Lemoine, M. (1995): Phys. Rev. D 52, 1955 
22. Copeland, E., Easther, R., Wands, D. (1997): Phys. Rev. D 56, 874

23. Abramovici, A., Althouse, W.E., Drever, R.W.P., Gursel, Y., Kawamura, S., Raab, F.J., Shoemaker, D., Sievers, L., Spero, R.E., Thorne, K.S., Vogt, R.E., Weiss, R., Whitcomb, S.E., Zucker, M.E. (1992): Science 256, 325;

Bradaschia, C. et al. (1990): Nucl. Instrum. Meth. A 289, 518

24. Durrer, R., Gasperini, M., Sakellariadou, M., Veneziano, G. (1998): Phys. Lett. B 436, 66 ;

(1999): Phys. Rev. D 59, 043511;

Melchiorri, A., Vernizzi, F., Durrer, R., Veneziano, G. (1999): Phys. Rev. Lett. 83, 4464

25. Veneziano, G. (1994): Strings, cosmology, ... and a particle. CERN-TH-7502-94

26. Gaillard, M., Murayama, H., Olive, K.A. (1995): Phys. Lett. B 355, 71;

Campbell, B.A., Gaillard, M., Murayama, H., Olive, K.A. (1999): Nucl. Phys. B 538, 351

27. Affleck, I., Dine, M. (1985): Nucl. Phys. B 249, 361

28. Damour, T. (1983): In Gravitational radiation, ed. by N. Deruelle, T. Piran, Amsterdam, pp. 59-144

29. Brady, P.R., Creighton, J.D.E., Thorne, K.S. (1998): Phys. Rev. D 58, 061501

30. Clark, J.P.A., Eardley, D.M. (1977) Astrophys. J. 215 311;

Blackburn, J.K., Detweiler, S. (1992): Phys. Rev. D 46, 2318;

Kidder, L.E., Will, C.M., Wiseman, A.G. (1992): Class. Quantum Grav. 9, L127;

(1993): Phys. Rev. D 47, 3281;

Wex, N., Schäfer, G. (1993): Class. Quantum Grav. 10, 2729;

Baumgarte, T.W., Cook, G.B., Scheel, M.A., Shapiro, S.L., Teukolsky, S.A. (1998): Phys. Rev. D 57 7299;

Baumgarte, T.W. (2000): Phys. Rev. D 62, 024018

31. Buonanno, A., Damour, T. (1999): Phys. Rev. D 59, 084006

32. Chandrasekhar, S., Detweiler, S. (1975): Proc. R. Soc. Lond. A 344, 441

33. Baker, J., Brugmann, B., Campanelli, M., Lousto, C.O., Takahashi, R.: gr-qc/0102037

34. Buonanno, A., Damour, T. (2000): Phys. Rev. D 62, 064015

35. Brézin, E., Itzykson, C., Zinn-Justin, J. (1970): Phys. Rev. D 1, 2349

36. Buonanno, A. (2000): Phys. Rev. D 62, 104022

37. Damour, T., Iyer, B.R., Sathyaprakash, B.S. (1998): Phys. Rev. D 57, 885; (2000): Phys. Rev. D 62, 084036

38. Ori, A., Thorne, K.S. (2000): Phys. Rev. D 62, 124022

39. Damour, T., Iyer, B.R., Sathyaprakash, B.S. (2001): Phys. Rev. D 63, 044023

40. Damour, T., Jaranowski, P., Schäfer, G. (2000): Phys. Rev. D 62, 084011 